\title{
Retrospective survey of human hydatidosis in Bahir Dar, north-western Ethiopia
}

\author{
N. Kebede, ${ }^{1}$ A. Mitiku ${ }^{2}$ and G. Tilahun ${ }^{7}$
}

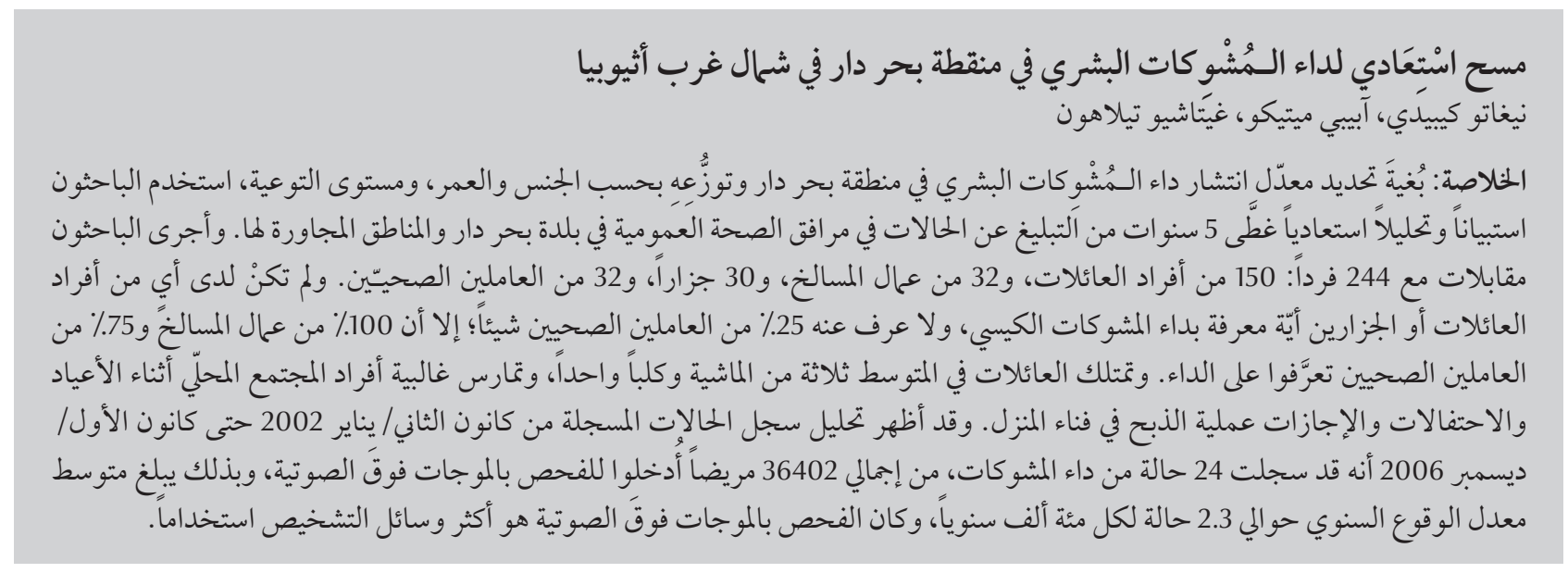

ABSTRACT To determine the prevalence of human hydatidosis in Bahir Dar and the sex and age distribution and awareness, we used a questionnaire and retrospective analysis covering 5 years of the case reports at public health institutions in Bahir Dar town and the surrounding areas. We interviewed 244 individuals: 150 households, 32 abattoir workers, 30 butchers and 32 health professionals. None of the householders or butchers knew about cystic echinococcosis, nor did $25 \%$ of the health professionals; however, $100 \%$ of the abattoir workers and $75 \%$ of the health professionals recognized it. The householders owned on average 3 livestock and 1 dog and during holidays, ceremonies and other feasts, the community mostly practised backyard slaughter. Case book analysis between January 2002 and December 2006 showed that out of the total of 36402 patients admitted for ultrasound examination, 24 hydatidosis cases were registered, giving a mean annual incidence rate of approximately 2.3 cases per 100000 per year. Ultrasound was the most frequently used diagnostic method.

\section{Étude rétrospective sur l'hydatidose humaine à Bahir Dar, au nord-ouest de l'Éthiopie}

RÉSUMÉ Afin de déterminer la prévalence de I'hydatidose humaine à Bahir Dar, sa répartition en fonction du sexe et de l'âge, ainsi que le degré de connaissance de la maladie, nous avons utilisé un questionnaire et une analyse rétrospective portant sur cinq années de rapports de cas recensés dans cette ville et ses environs. Nous avons interrogé 244 personnes : 150 chefs de famille, 32 ouvriers d'abattoir, 30 bouchers et 32 professionnels de santé. Aucun chef de famille, ni aucun boucher n'avait entendu parler de l'échinococcose kystique, mais $100 \%$ des ouvriers d'abattoir et $75 \%$ des professionnels de santé la connaissaient. Les chefs de famille possédaient en moyenne trois animaux d'élevage et un chien. Durant les jours de congés, les cérémonies et autres fêtes, la plupart des membres de la communauté pratiquaient l'abattage domestique. L'analyse des registres entre janvier 2002 et décembre 2006 a révélé que, sur un total de 36402 patients admis pour une échographie, 24 cas d'hydatidose avaient été enregistrés, portant le taux d'incidence annuelle moyen à environ 2,3 cas pour 100000. L'échographie était la méthode de diagnostic la plus fréquemment employée.

${ }^{7}$ Aklilu Lemma Institute of Pathobiology, Addis Ababa University, Addis Ababa, Ethiopia (Correspondence to N. Kebede: knigatu2003@yahoo. com,nigatukebede@yahoo.com).

${ }^{2}$ Amhara National Regional State Bureau of Agriculture and Rural Development, Bahir Dar Regional Veterinary Laboratory, Bahir Dar, Ethiopia. Received: 05/11/08; accepted: 19/02/09 


\section{Introduction}

Cystic echinococcosis (CE) (hydatidosis), caused by the larval stage of Echinococcus granulosus, is recognized as being one of the world's major zoonoses $[1,2]$. The distribution of hydatidosis is normally associated with underdeveloped countries, especially in rural communities where humans maintain close contact with dogs and various domestic animals, which may act as intermediate hosts [2].

Dogs are the primary definitive hosts for the parasite, with livestock acting as intermediate hosts and humans as aberrant intermediate hosts. Humans become infected by the ingestion of eggs passed in the faeces of dogs [3]. The outcome of infection in humans is hydatid cyst development in the lung, liver or other organs [4]. This causes severe disease and death in humans and results in economic loss from treatment costs, lost wages and livestockassociated production losses [4].

E. granulosus has a cosmopolitan distribution [5] and CE is a public health problem in Asia, the Mediterranean, South America and Africa. Previous reports describe the endemic occurrence of human cases of CE in North African countries bordering the Mediterranean [6] and in sub-Saharan Africa [7].

In Ethiopia, the situation of CE in humans is not well documented and explored. In southern parts of Ethiopia prevalence rates of $1.6 \%$ and $0.5 \%$ have been reported [5]. Hence, the aim of the current study was to determine the prevalence, sex and age distribution and awareness information of human hydatidosis in Bahir Dar.

\section{Methods}

\section{Study area}

The study was conducted in Bahir Dar town in Amhara National Regional State in north-western Ethiopia. Bahir Dar is $565 \mathrm{~km}$ from Addis Ababa and has 1 public hospital, 1 health centre and 3 higher private clinics. The population of the town is about 205000 [8].

\section{Study design}

A questionnaire and retrospective hos$\mathrm{pital} /$ clinical data survey were the tools used in the study. A structured questionnaire was prepared in the local language and pilot tested on 10 individuals from the same population to assess performance. The questionnaire gathered information on the extent of awareness of $\mathrm{CE}$, control measures taken and related factors. The participants were selected based on their occupation using simple random sampling. All 32 meat inspectors were selected, plus 50\% (30) of the butchers, $50 \%$ (total 32) of the health professionals per institute and $10 \%$ (150) of the householders. We collected retrospective data on ultrasound examinations which had been carried out for different purposes during the period January 2002-December 2006 from Felegehiowt Hospital and Kidane Mehiret, Gambi and Eyerusalem health services. Data on human hydatid infection were identified, and the mean annual incidence of human hydatidosis was determined.

\section{Data analysis}

Percentages prevalence was determined and analysis of variance (ANOVA) carried out to measure the differences by age-group and the odds ratios for differences according to sex. In all the analyses, confidence level was 95\% and significance level was 5\%.

\section{Ethical approval}

The study was conducted after the objectives were explained to those completing the questionnaire and written agreement was obtained from them.

\section{Results}

\section{Questionnaire survey}

None of the households interviewed in the study area knew about CE. They owned on average 3 livestock and 1 dog. The animals were kept for draught power and as a source of income. The animals grazed communally where livestock and humans had frequent contact with dogs and their excreta. During holidays, ceremonies and other feasts, the community mostly practised homestead/backyard slaughter; during slaughter, meat inspection is not conducted and the offal is often given to pets or disposed of in the backyard. In $10.7 \%$ of the households, the dogs were treated with traditional preparations at their puppy stage only; other than this, treatment with commercial drugs was not practised at all. The dogs were kept for guarding the homestead and hunting.

All (100\%) of the 32 abattoir workers we interviewed in Bahir Dar recognized hydatid cyst and the organs frequently affected without knowing the name of the disease. When an organ affected by hydatid cyst was encountered, it was totally condemned.

Only $75 \%$ of the 32 health professionals working in public health institutions were aware of $\mathrm{CE}$.

Among the 60 butchers present in Bahir Dar town, 30 were interviewed; none $(0 \%)$ of whom were aware of the disease.

Ultrasound was the most frequently used diagnostic method and up to 2 cases were encountered per year. However, for various reasons the professionals had never tried to educate people or create awareness about the disease.

\section{Retrospective hospital and clinical case-book survey}

From a total of 36402 patients admitted for ultrasound examination, about 24 human CE cases were registered between January 2002 and December 2006, 8 were from Felegehiwot Hospital, 12 from Gambi Clinic, 3 from Kidanemeheret Clinic and 1 from Eyerusalem Clinic. Gambi Clinic had the highest rate for hydatidosis cases among the 19160 patients admitted (52.6\% of 


\begin{tabular}{|c|c|c|c|c|c|c|c|}
\hline \multirow[t]{2}{*}{ Hospital/clinic \& patient type } & \multicolumn{7}{|c|}{ No. patients admitted } \\
\hline & 2002 & 2003 & 2004 & 2005 & 2006 & Total & $\%$ \\
\hline \multicolumn{8}{|l|}{ Felegehiwot Hospital } \\
\hline Admitted & 624 & 2687 & 2822 & 680 & 793 & 7606 & 20.9 \\
\hline Cases & - & 4 & 4 & - & - & 8 & 33.3 \\
\hline \multicolumn{8}{|l|}{ Gambi Clinic } \\
\hline Admitted & - & 2803 & 3780 & 8919 & 3658 & 19160 & 52.6 \\
\hline Cases & & 2 & 2 & 6 & 2 & 12 & 50.0 \\
\hline \multicolumn{8}{|l|}{ Kidanemehiret C linic } \\
\hline Admitted & - & - & 712 & 3637 & 1441 & 5790 & 15.9 \\
\hline Cases & - & - & - & 3 & - & 3 & 12.5 \\
\hline \multicolumn{8}{|l|}{ Eyerusalem Clinic } \\
\hline Admitted & - & - & 875 & 2280 & 763 & 3846 & 10.6 \\
\hline Cases & - & - & - & 1 & - & 1 & 4.2 \\
\hline \multicolumn{8}{|l|}{ Total } \\
\hline Admitted & 624 & 5490 & 8189 & 15444 & 6655 & 36402 & 100.0 \\
\hline Cases & 0 & 6 & 6 & 10 & 2 & 24 & 100.0 \\
\hline Prevalence (\%) & 0 & 0.11 & 0.07 & 0.06 & 0.03 & 0.066 & $0.066^{\mathrm{a}}$ \\
\hline
\end{tabular}

${ }^{a} 0.066=2.3$ cases per 100000 population of the study area

the total examined) with 12 cases, about $50 \%$ of the total recorded in this study (Table 1).

Age and sex groups of human hydatid cases are described in Tables 2 and 3. Females were much more likely to be infected than males, and the highest prevalence was in the age group 21-30 years.

Hydatid cyst was encountered most commonly in the liver $79.2 \%$ (19 of the 24 cases registered during ultrasound examination) and less frequently in the spleen $20.8 \%$ (5 cases).
Only 2 patients had surgical intervention and none received chemotherapy. During the last 5 observation years, major surgical operations were only performed at Felegehiwot Hospital.

According to the surgical case recording book at the hospital, 3203 patients (2229 males and $974 \mathrm{fe}$ males) with a variety of health problems underwent surgical intervention. Only 2 of these patients $(0.06 \%$, both females) were recorded as hydatid cyst cases.

Table 2 Age distribution of human hydatid cases in public health institutions in Bahir Dar, January 2002-December 2006

\begin{tabular}{lccc} 
Age (years) & Total examined & \multicolumn{2}{c}{ Infected } \\
& \multicolumn{3}{c}{ No. } \\
\hline 10 & 6524 & - & \\
$11-20$ & 7332 & 2 & 0.03 \\
$21-30$ & 6898 & 13 & 0.19 \\
$31-40$ & 6912 & 5 & 0.07 \\
$>40$ & 8736 & 4 & 0.05 \\
Total & 36402 & 24 & 0.07 \\
\hline
\end{tabular}

$F=5.540$, degrees of freedom $=4, P=0.001$.

\section{Discussion}

The culture and tradition of inhabitants in the study area favour the keeping of dogs in periurban areas (urban and rural households) often in close association with the family and farm animals. Almost all cattle owners, shepherds and urban dwellers keep at least 1 dog to safeguard their properties from wild carnivores and thieves. These and other socioeconomic realities in the areas studied are considered to be conducive to the maintenance and further propagation of hydatid disease.

CE is not currently a well known medical condition, nor it considered to be of public health importance; it is not a notifiable disease and there are limited surveillance data available in humans in the country. However, in the residents in the study area, the annual incidence rate was approximately 2.3 cases per 100000 per year, calculated based on the catchments of the hospital. In 2 community-based surveys conducted in southern Ethiopia in 1987 and 1996, 1997 and 3224 participants respectively 


\begin{tabular}{lcccccc}
\hline \multicolumn{6}{l}{ Table 3 Sex distribution of human hydatid cases in public health institutions in Bahir Dar, January $\mathbf{2 0 0 2}$ to December 2006} \\
\hline Sex & $\begin{array}{c}\text { Total no. } \\
\text { examined }\end{array}$ & No. Infected & $\%$ & Odds ratio & $\mathbf{9 5 \%} \mathbf{C l}$ & $\boldsymbol{P}$-value \\
Female & 19298 & 20 & 0.10 & 4.435 & $1.516-12.978$ & 0.02 \\
Male & 17104 & 4 & 0.02 & 0.226 & $0.077-0.659$ & 0.2 \\
Total & 36402 & 24 & 0.07 & & & \\
\hline
\end{tabular}

$C I=$ confidence interval.

were screened with ultrasound: prevalence rates were $1.6 \%$ ( 31 cases) and $0.5 \%$ (16 cases) respectively [5]. The average annual incidence in humans per 100000 has been reported in a number of studies, e.g. 2.2 in Portugal [9], 3.3 in Bulgaria [10], 3.4 in Greece [11], 3.6-15.8 in Morocco [12] and 3.4-4.6 in Algeria [13]. Our findings 2.3 per 100000 per year, was comparable with these studies, and showed that $\mathrm{CE}$ is a zoonosis of importance in Bahir Dar. This may be because of the low public awareness, backyard slaughtering practices, poor control measures, the presence of a large number of stray dogs and poor infrastructure that contributed to human infection in this area.

In the study area, the number of patients admitted and the number of hydatid cases were unexpectedly high at Gambi private clinic. This may be related to the preference of patients for that clinic due to the relatively superior disease diagnosis. The finding that all hydatid cases were in the age groups over 10 years implies that both young people and adults are susceptible to infection. A higher incidence rate was seen in females than males; this may be due to the fact that females had more contact time with dogs than males around the home

Retrospective hospital and clinic survey data on human CE gave an estimate of the prevalence of hydatidosis in the study area. The infection is asymptomatic with an extended incubation period, and there is a clinical similarity with other diseases. ths study area is lacking in modern diagnostic facilities, and there is inability to afford modern treatment by the most vulnerable section of society. However, despite such limitations, careful examinations of hospital/clinic records provided a useful indication of infection rates. This, when done continuously over many years, may detect changes in the trend and incidence of infection.

It is therefore concluded that $\mathrm{CE}$ is a disease of considerable importance for public health. In view of the results of this retrospective survey, and considering the nature of the disease, the actual natural, social, cultural and behavioural situation in Bahir Dar and the surrounding areas are conducive to the maintenance of a high level of infection and spread of the disease between animals and humans. Improving public awareness (education), avoidance of stray dogs, meat control legislation and proper disposal of condemned organs are recommended to mitigate the effect of the disease on public health.

\section{Acknowledgements}

The authors would like to acknowledge staff members of Felegehiowt Hospital and Kidane Mehiret, Gambi and Eyerusalem health services in Bahir Dar, and the abattoir workers and butchers.

Financial support was obtained from the Research and Graduate Studies Department of Addis Ababa University and Amhara Regional Agricultural Research Institute (ARARI).

\section{References}

1. Eckert J, Deplazes P. Biological, epidemiological, and clinical aspects of echinococcosis, a zoonosis of increasing concern. Clinical Microbiology Reviews, 2004, 17(1):107-135.

2. Torgerson PR, Budke C. Echinococcosis - an international public health challenge. Research in Veterinary Science, 2003, 74:191-202.

3. Budke CM, Deplazes P, Torgerson PR. Global socioeconomic impact of cystic echinococcosis. Emerging Infectious Diseases, 2006, 12(2):296-303.

4. Jenkins DJ, Romig T, Thompson RCA. Emergence/ re-emergence of Echinococcus spp.a global update. International Journal for Parasitology, 2005, 35:1205-1219.

5. Eckert J et al. WHO/OIE manual in echinococcosis in humans and animals. Geographic distribution and prevalence. Paris, World
Health Organization and World Organisation for Animal Health, 2002:101-143.

6. Magambo J, Njoroge E, Zeyhle E. Epidemiology and control of echinococcosis in sub-Saharan Africa. Parasitology International, 2006, 155:S193-S195.

7. Shambesh MA et al. An extensive ultrasound and serologic study to investigate the prevalence of human cystic echinococcosis in northern Libya. American Journal of Tropical Medicine and Hygiene, 1999, 60:462-468.

8. Livestock resource development and animal health department annual report. Bahir Dar, Ethiopia, Bureau of Agriculture and Rural Development (BoARD), 2006:1-8.

9. Battelli G. Socioeconomic impact of CE. WHO Mediterranean Zoonoses Control Centre Information Circular, 2003, 57:1020-1378. 
10. Todorov T, Boeva V. Epidemiology of echinococcosis in Bulgaria-a comparative study. Inernational Archive for Hidatidosis, 1997, 32:232-233.

11. Economides P, Thrasou K. Echinococcosis/hydatidosis and program for its control in the Mediterranean countries. International Archive for Hidatidosis, 1999, 33:63-83.

12. El Idrissi et al. Retrospective survey for surgical cases of CE in Morocco (1980-1992). Provo, Utah, United States of America, Brigham Young University, 1997:194-222.
13. Shambesh MK. Human CE in North Africa. In: Andersen FL, Ouhelli H, Kachani M, eds. Compendium on CE in Africa and in Middle Eastern countries with special reference to Morocco. Provo, Utah, United States of America, Brigham Young University, 1997:223-244.

\section{Global Early Warning System for Major Animal Diseases, including Zoonoses (GLEWS)}

Disease emergence and spread do not respect geographical boundaries, and animals are often implicated as the source of human infection. Zoonotic disease management therefore requires an integrated approach that involves different sectors; mainly human, animal and food. Efficient early warning and forecasting of zoonotic disease trends through functional surveillance systems is key to effective containment and control. Early intervention during a disease epidemic often leads to better outcomes with reduced disease burden and associated economic impact.

GLEWS is a joint system that builds on the added value of combining \& coordinating alert mechanisms of the World Health Organization (WHO), the Food and Agriculture Organization of the United Nations (FAO) and the World Organisation for Animal Health (OIE), while linking networks from the international community and stakeholders, to assist in prediction, prevention and control of zoonotic disease threats through sharing of information, epidemiological and risk analysis.

More information about GLEWS can be found at: http://www.glews.net 